Es ist erstaunlich, wieviel wirksamer ein so hergerichteter Trockenapparat ist, was folgendes Beispiel lehren mag:

In zwei gewöhnlichen Exsiccatoren von ganz gleicher Grösse wurden Uhrgläser mit je 10 ec Wasser aufgestellt und zwar so, dass sich in dem einen Apparat in möglichst gleicherEntfernung concentrirte Schwefelsäure über, in dem andern unter dem Uhrglase befand. Das Resultat des Versuches war, dass das Wasser in dem Exsiccator mit der gebräuchlichen Anordnung 9 Tage brauchte, um zu verdunsten, während es in dem Apparat, wo das Trockenmittel oberhalb angebracht wurde, nach 3 Tagen verschwunden war.

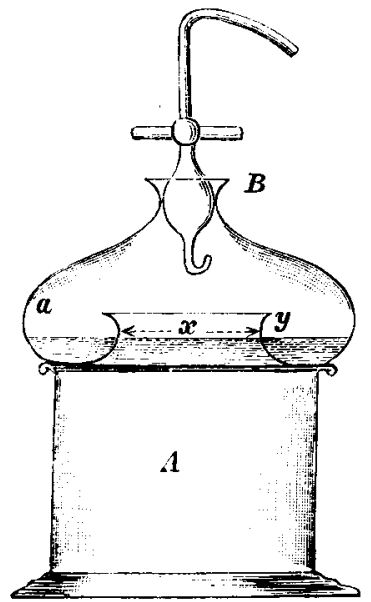

Fig. 113.

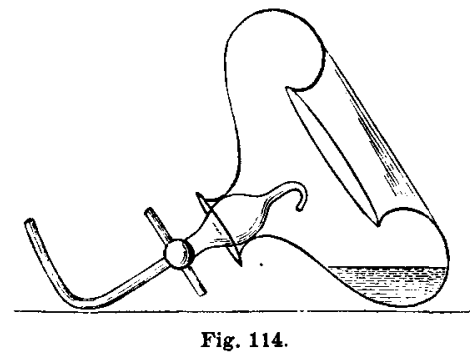

Nach mehrfachen Versuchen, einen geeigneten Apparat kerzustellen, der diesen Gesichtspunkten Rechnung trägt, ist es mir gelungen, eine Form zu finden, welche sich als praktisch erwiesen hat.

Der neue Exsiccator Fig. 113 u. 114 besteht aus einem cylindrischen Glas $A$ zur Aufnahme der zu trocknenden Gegenstände und dem Deckel $B$. Der Deckel $B$ hat die Form der bekannten Fliegenfänger; die Schwefelsäure befindet sich in dem Ringraum $a$, ein eingeschliffener Stöpselhahn gestattet, den Apparat luftleer zu pumpen. Der Deckel bietet durch seine eigenthümliche Form den Vortheil, dass man ihn nach dem Öffnen des Apparates weglegen kann, ohne den unteren Rand aufstellen zu müssen, wodurch leicht eine Verunreinigung der Schlifffächen durch Sandkörner herbeigeführt werden könnte. Bringt man nämlich den Deckel in die Stellung, welche Fig. 114 zeigt, so sammelt sich die Schwefelsäure in dem Bauche des Deckels, ohne dass auch nur ein Tropfen davon herausfliesst. Es ist zweckmässig, die Öffnung $a$ möglichst weit zu machen und den inneren Rand bei $y$ etwas nach innen zu biegen.

Greiner \& Friedrichs in Stützerbach haben mir sehr schöne derartige Exsiccatoren geliefert.

\section{Über das Auftreten von Cyaniden bei der Zinkgewinnung.}

$$
\text { Von }
$$

\section{Edmund Jensch.}

Bei dem Hochofenprocess ist bekanntlich die Bildung ron Kaliumcyanid eine alltägliche Erscheinung, bei dem Zinkhüttenprocesse jedoch ist trotz der sehr ähnlichen Betriebsverhältnisse die Bildung von Cyaniden eine so seltene, dass es dem Verfasser im Verlaufe mehrerer Jahre erst einmal gelungen ist, das Auftreten von Cyanverbindungen festzustellen. Es sei daher eine kurze Mittheilung über diese Beobachtung gestattet.

Die Aufmerksamkeit wurde dadurch auf diese Verbindungen gelenkt, dass die aus den Zinkstaubfängern (Allongen) entweichenden Abgase eines einzigen Muffelpaares für kurze Zeit - etwa 10 bis 15 Minuten lang - den bekannten Geruch aufwiesen und ebenso der während eines 20 stündigen Destillationsprocesses in diesen Vorlagen (Allongen) angesammelte oxydhaltige Zinkstaub deutlich denselben Geruch zeigte und auch für längere Zeit beim Aufbewahren im verschlossenen Gefässe behielt. Die Erzbeschickung dieses Muffelpaares wich indess von derjenigen der anderen Muffeln in keiner Weise ab, so dass eine Erklärung über die Entstehungsweise dieser Cyanverbindungen nicht gegeben werden kann.

Von sichtlichem Interesse war nun die Frage, in welcher Form bez. mit welchem Metalle vereinigt das Cyan hier auftrat. Angesichts der Thatsache, dass das Kaliumcyanid ungemein feuerbeständig ist und erst bei höherer Rothglut schmilzt, lag die Vermuthung nahe, dass diese Verbindung bereits in der Muffel entstanden und dann 
einfach in die Vorlagen überdestillirt sei. In diesem Falle musste also der Zinkstaub cyankaliumbaltig sein. Andererseits konnte sich aber auch eine bei dieser Temperatur flüchtige Cyanzinkverbindung schon in der Muffel gebildet haben, die ebenfalls in den kühleren Vorlagen sich wieder verdichtete. Oder aber die Cyanbildung vollzog sich überhaupt erst in den kühleren Theilen, namentlich in den Zinkstaubrorlagen, in die ja schon ein reichlicher Zutritt von atmosphärischer Luft erfolgt. In diesem Falle würde die Entstehung der Cyanide zurückzuführen sein auf die unmittelbare Einwirkung von Stickstoff auf das Gemisch von Dämpfen metallischen Zinks mit Kohlenoxydulgas und nach folgender Gleichung stattfinden:

$3 \mathrm{Zn}+2 \mathrm{CO}+\mathrm{N}_{2}=\mathrm{Zn}(\mathrm{CN})_{2}+2 \mathrm{ZnO}$.

Áusserdem lag aber auch noch die Möglichkeit vor, dass das fein vertheilte Zinkoxyd bei Gegenwart der Metalldämpfe auf überdestillirtes Kaliumcyanid eingewirkt und nachstehende Umsetzung herbeigeführt hätte: $\mathrm{ZnO}+2 \mathrm{~K}(\mathrm{CN})_{2}=\mathrm{Zn}(\mathrm{CN})_{2}+\mathrm{K}_{2} \mathrm{O}$.

Angesichts der grossen Mengen staubförmigen Zink und Zinkoxydes gegenüber den Spuren von Cyan konnte dieser Nachweis bez. die Trennung etwaiger Alkalicyanide von den entsprechenden Metallverbindungen nicht mittels Wassers bewirkt werden. Da aber Ätzkali sich auch in wasserfreiem Alkohol leicht löst, die Cyanide, $\mathrm{Zn}$ und $\mathrm{Zn} \mathrm{O}$ dagegen darin unlöslich sind, so sollte durch Auslaugen dieses Zinkstaubes mittels des letzgenannten Lösungsmittels dargethan werden, ob eine Umsetzung von Kaliumcyanid mit Zinkoxyd eingetreten wäre. In Folge dessen wurden $300 \mathrm{~g}$ Zinkstaub der Auslaugung unterworfen, Kaliumoxyd war jedoch in dem abgezogenen Alkohol nicht nachweisbar, auch konnte derselbe ohne Rückstand überdestillirt werden.

Eine Umsetzung nach der letzten Gleichung hatte demgemäss nicht stattgefunden. Da ferner der ausgelaugte Rückstand, wie eine Flammenreaction ergab, alkalifrei war, so konnte auch nicht Kaliumcyanid unmittelbar aus der Muffel in die Vorlage verdampft sein - die vorhandene Cyanverbindung musste also zinkhaltig sein.

Bei längerem Kochen dieses Zinkstaubes mit cyanfreier Natronlauge konnte in dem Filtrat mittels Silbernitrats deutlich Cyan nachgewiesen werden. Da das Material frei von Erdalkalien war und man das Blei in Form des Sulfats erhielt, so ist also das Vorhandensein eines Zinkcyanids anzunehmen, doch bleibt die Entstehung desselben noch unaufgeklärt.
Wirkung der Schwefelalkalien auf die Lösungen der Metalle der Eisengruppe.

\author{
Vorläufige Mittheilung \\ von \\ Prof. Dr. L. L. de Koninck \\ und \\ M. Ledent, \\ Candidat Scient.
}

In den Lehrbüchern der analytischen Chemie, von H. Rose's Handbuchs ersten Ausgaben an bis zu den allerneuesten, wird angegeben, dass das Schwefelammon in den Lösungen von Eisen, Mangan, Zink, Nickel und Kobalt einen Niederschlag von Schwefelmetallen hervorbringt; die einzigen angeführten Eigenthümlichkeiten dieser Reactionen lauten folgendermaassen:

1. Es bleibt bisweilen etwas Schwefeleisen in Suspension; in diesem Falle ist die Flüssigkeit grün, aber nach längerem Stehen verschwindet diese Färbung, und die Fällung des Eisensulfürs wird vollständig.

2. Die Fällung des Mangans ist nicht vollständig; Spuren dieses Metalles findet man im rom Niederschlage getrennten Filtrate, selbst dann, wenn das Filtrat durchaus klar ist.

3. Das Schwefelnickel ist oft in einem Übermaasse von Schwefelammonium etwas löslich; die Auflösung ist braun. Bei den Kobaltsalzen kommt diese Erscheinung nicht vor.

In Betreff der Wirkung des Schwefelkaliums und Schwefelnatriums wird gewöhnlich nichts erwähnt, oder, wenn davon die Rede ist, vermischt man diese Reagentien mit dem - Schwefelammon unter der Gemeinbezeichnung von Schwefelalkalien.

Wir kennen keine Abhandlung über die Wirkung der fixen Schwefelalkalien auf die ebengenannten Metallsalze.

Die einzigen, unseres Wissens diesbezüglichen Arbeiten über die Wirkung des Schwefelammons sind folgende: erstens eine schon alte und sehr oberfächliche Notiz von Terreil ${ }^{1}$ ), nach welcher, wenn Ammoniaksalze und Ammoniak im Überschuss vorhanden sind, die Zink-, Mangan-, Nickel- und Kobaltsalze nicht oder doch sebr unvollständig von Schwefelammon gefällt werden; die Nickel- bez. Kobaltlösungen wären beide mahagonibraun $^{2}$ ). Nach Terreil wäre im Gegentheil die Fällung der Ferrosalze (der

1) Compt. r. 45 S. 652 (1857).

2) War das angewandte Kobaltsalz vollständig nickelfrei? 